\title{
The SYNCAN project: goals, set-up, first results and settings of the human intervention study
}

\author{
Jan Van Loo ${ }^{1 *}$, Yvonne Clune ${ }^{2}$, Mary Bennett ${ }^{2}$ and John Kevin Collins ${ }^{2}$ \\ ${ }^{1}$ Orafti, Aandorenstraat 1, B3300 Tienen, Belgium \\ ${ }^{2}$ Departments of Microbiology and Medicine, Alimentary Pharmabiotic Centre, University College Cork, Cork, Ireland
}

\begin{abstract}
Experimental evidence on the anticancer properties of dietary prebiotics such as chicory inulin and oligofructose and dietary probiotics has accumulated in recent years. Various experimental models ranging from chemoprevention studies, tumour implantation models to genetically modified mice models, etc. have systematically shown the protective effects of these food ingredients. In some studies it appeared that synbiotics (combination of pre- and probiotics) exerted synergistic activity against processes of carcinogenesis. The logical next step in research was to find out if these observations also would be valid for human volunteers. This was the principal goal of the EU-sponsored SYNCAN project (QLK1-1999-346) which involved the integration of an in vitro study to select the most suitable synbiotic preparation, the application of this synbiotic in an in vivo rat model of chemically induced colon cancer, and, as the heart of the project, the investigation of the synbiotic effects in a human intervention study. The in vitro tests consisted of fermentation studies where the interaction of pre- and probiotics was studied. Cell-free supernatants were generated from various synbiotic combinations fermented by faecal slurry, which were then used to optimise a series of bioassays. In the rat study the anticarcinogenic effect of prebiotics and synbiotics but not of probiotics was demonstrated. Using tissue samples generated in this model, attempts were made to gain a better insight into the mechanisms underlying cancer development. The human intervention study consisted of two groups of volunteers. One group was composed of people at high risk (polypectomised subjects) for colon cancer and the other of volunteers (colon cancer subjects) who had previously undergone 'curative resection' for colon cancer but were not currently receiving treatment. The present paper describes the experimental design of the SYNCAN study, and demonstrates a functional effect of the synbiotic preparation (probiotic survival during gastrointestinal transit and modification of the intestinal flora). Detailed experimental outcome of the human intervention study will be reported elsewhere.
\end{abstract}

Colon cancer: Lactobacillus: Bifidobacterium: Inulin: Oligofructose: Man

Prebiotics are food ingredients that cannot be degraded by intestinal and pancreatic digestive enzymes, and as such are completely available for fermentation by the intestinal flora. An additional requirement for a product to be a prebiotic is that it has to be selectively fermented, so that the composition of the intestinal flora improves (a relative increase of saccharolytic flora often accompanied by a decrease in proteolytic fermentation; bifidobacteria and lactic acid bacteria are indicator organisms). The modification in both the composition and the metabolic activity of the intestinal flora is the basis of the nutritional benefits of prebiotics. These beneficial effects include improving bowel habit and mineral absorption, modulating lipid metabolism and the immune system, and retarding the process of carcinogenesis.

Salminen et al. (1999) have defined probiotics as 'microbial cell preparations or components of microbial cells that have a beneficial effect on the health and well-being of the host.' Although not always essential, it may be desirable that probiotics are viable in order to exert their beneficial nutritional effects during their passage through the intestinal tract. Additionally, they need to be able to survive exposure to gastric juice and bile acids in the upper gastrointestinal tract. Some of the beneficial activities associated with the consumption of probiotics include immune stimulation, reduced incidence of intestinal infection and in some cases an inhibitory effect on carcinogenesis.
Over the past decade, a solid basis of evidence for the anticarcinogenic effects of prebiotics and probiotics has been built up. The anticancer properties of prebiotics or probiotics have been shown by means of various models.

In chemoprevention models, laboratory animals are challenged by injection of a carcinogenic chemical such as azoxymethane (AOM) or 1,2-dimethylhydrazine to induce colon cancer, or 4-methyl nitrosourea to induce breast cancer in female test animals. Typically, animals are administered either a control diet or a diet containing the prebiotic substances (e.g. inulin, oligofructose $5-10 \%$ added to the diet), or the probiotic bacteria $\left(10^{9}-10^{10} \mathrm{cfu} / \mathrm{d}\right)$ or a combination of the two, described as a synbiotic. The main end point of these experiments is the formation of preneoplastic lesions (after 2 months) or tumours (after 35-52 weeks). In other cases, the growth of previously implanted tumours can be monitored as a function of time (volume is measured with a Vernier calliper). From such experiments there is consistent evidence that inulintype fructans (Reddy et al. 1997; Rao, 2001; Verghese et al. 2002), some probiotics (Kulkarni \& Reddy, 1994; Gallaher et al. 1996) and certain synbiotic combinations (Rowland et al. 1998; Bolognani et al. 2001) are able to significantly reduce (up to $80 \%$ ) the number of preneoplastic lesions (aberrant crypt foci or mucin-depleted foci), as well as the number of tumours. Synbiotics are not the only combinations to have shown improved 
anticancer activity as it was also shown that a combination of prebiotics which differently influence colonic fermentation (i.e. oligofructose and long-chain inulin known as oligofructoseenriched inulin or Synergy ${ }^{\circledR}$ ) showed a stronger anticancer potential (Verghese et al. 2003; Van Loo, 2004). A significant retardation of the development of chemically induced mammary tumours has also been reported with inulin or oligofructose (Taper \& Roberfroid, 1999).

Another carcinogenesis model is the tumour implantation model where aggressive and invasive tumour cells are implanted either in the muscle or in the peritoneum of mice. The animals are either fed control or prebiotic inulin-type fructans supplemented food 1-2 weeks before the tumour implantation and mortality curves are monitored. Histological evaluation is used to determine the metastatic potential and the size and type of tumour. Dietary inulin and oligofructose were shown to significantly reduce the development of the tumours, with, as a consequence, an increase in life span (Taper et al. 1998). The tumours that still developed were less invasive in the prebiotic fed group (Taper \& Roberfroid, 2000).

Genetically predetermined models (APC ${ }^{\text {min }}$ mice) spontaneously develop tumours, mainly in the small intestine but also in the colon. Experiments with this model have shown that consumption of oligofructose reduces the number of colonic tumours (Pierre et al. 1997). A new Western style diet designed by Lipkin and supplemented with oligofructose and inulin was fed to $\mathrm{APC}^{\mathrm{min}}$ mice and it has been reported that the mice developed significantly less tumours in both the small intestine and in the colon (M Lipkin, unpublished results). Probiotics have also been shown to reduce tumour incidence in genetic models. Consumption of Lactobacillus salivarius ssp. salivarius UCC118 in IL-10 knockout mice (colitis model) was associated with a trend towards reduced tumour development and attenuation of gastrointestinal inflammation. Gastrointestinal neoplastic transformation was evident in $50 \%$ of mice from the placebo group while only $10 \%$ of the probiotic group demonstrated any evidence of neoplastic change (O'Mahony et al. 2001).

These and other similar experiments show an interesting potential for prebiotics and/or probiotics. As it is difficult to extrapolate results from experimental rodent models to man, it was necessary to verify if these food ingredients also have a potential to reduce risk for colon cancer in human volunteers. The SYNCAN project was designed to investigate whether or not this is the case (Van Loo \& Jonkers, 2001). In the present paper, the strategy of the SYNCAN project is laid out. Published data from experimental models are summarised and the setting of the human intervention study is explained. The experimental outcome and interpretation of the human intervention study will be published elsewhere.

\section{Materials and methods}

\section{Prebiotics and probiotics}

The prebiotic used in the SYNCAN project was a combination of fructan fractions with different fermentation characteristics known as oligofructose-enriched inulin or 'Synergy1' (Raftilose ${ }^{\circledR}$ Synergy1 from Orafti, Belgium). One fraction was composed of linear $\beta(2-1)$ fructans with a degree of polymerisation (DP) ranging between 10 and 65 (average DP was 25) and the other fraction contained similar molecules but with chain length varying between 2 and 8 (average DP is 4). The latter molecules are rapidly fermented by the colonic flora leading to modification of the composition of the intestinal flora in a proximal part of the colon. The longer chains are typically fermented at a slower rate, but still in a selective way. This longer chain fraction has the ability to maintain the metabolic activity of the improved flora for a longer period of time, or spatially, in more distal parts of the intestine. In the experimental models it was shown that this mixture of inulin-type fructans could reduce the incidence of aberrant crypt foci significantly more than its individual components alone (Verghese et al. 2003). It was also thought that a cocktail of probiotics would be more efficient than a single strain. From a practical point of view it was decided to take existing commercial strains with a history of anticancer properties in experimental models. The selected strains were Lactobacillus rhamnosus GG called 'LGG' (from Valio, Finland) and Bifidobacterium lactis Bb12 called 'Bb12' (from Christian Hansen Labs, Sweden).

\section{Results}

\section{In vitro fermentation studies}

The in vitro studies included fermentation of various combinations of the prebiotics, probiotics and synbiotics either alone (pure culture of bacteria) or in the presence of faecal slurry as inoculum. Control fermentations (prebiotics replaced by glucose and no added probiotics) were included. Those fermentations were performed either as batch fermentations or as three-stage continuous fermentations mimicking the three compartments of the colon (McBain \& Macfarlane, 1997). All fermentations were carried out in anaerobic conditions and with controlled $\mathrm{pH}$ and temperature.

Cell-free supernatants of such fermentation broths were applied to two colon cancer cell lines (HT29 and CaCo-2) and cellular parameters of survival, differentiation, tumour progression and invasive growth were monitored. Interestingly, samples that originated from fermentations with prebiotics alone or synbiotics were significantly less cytotoxic than control fermentation supernatant (based on glucose as C source). The observed lower toxicity could be correlated with relative higher butyrate concentration in the prebiotic fermentations. Samples originating from the threestage gut model showed that the third compartment, which had the highest concentration of butyrate, also had the lowest cytotoxic potential. These data thus support the hypothesis that effects of oligofructose-enriched inulin on colon cells could be due to an altered microflora, producing higher amounts of butyrate. However, it is likely that this is not the only parameter that can explain the observations. Further research involving bacteria-host interactions might give more insight in these matters. This in vitro part of the SYNCAN project gives a rational support to the prebiotic and/or synbiotic approach to reduce the risk of colon cancer development (Klinder et al. 2003).

\section{In vivo animal study}

The in vivo animal study was designed to compare the effects of the probiotics, the prebiotics and the synbiotic combination in a model of carcinogenesis. As intestinal and immunological tissues are more readily available than in human volunteers, this experimental approach was also taken to help elucidate the possible mechanisms of action of prebiotics, probiotics and synbiotics.

Four groups of twenty-five rats were fed control diet, prebiotic diet (control diet $+10 \%$ Synergy 1 ), probiotic diet 
(control diet $+10^{8} \mathrm{cfu} / \mathrm{g} \mathrm{Bb} 12$ and LGG) or the synbiotic diet (Fig. 1). After $10 \mathrm{~d}$ of adaptation to the diets the rats were injected twice with $15 \mathrm{mg} \mathrm{AOM} / \mathrm{kg}$ body weight with 1 -week interval. Tumour incidence was determined after 31 weeks. The results show that the prebiotic and the synbiotic diets, but not the probiotic diet, significantly reduced the tumour incidence in the colon. The synbiotic-fed group had fewer tumour-bearing rats than the two other groups (prebiotic and probiotic). In the prebiotic- and synbiotic-fed groups, the concentration of SCFA was almost twice that in the control and the probiotic groups; the molar ratio of butyrate also was significantly higher in the prebioticand synbiotic-fed groups (Femia et al. 2002). This was consistent with the possible protective effect of butyrate observed in the in vitro part of the project.

Faecal water (FW), which is the aqueous phase of faeces, contains most of the free reactive and soluble factors thought to interact with the mucosa in vivo. The cytotoxic and genotoxic potential of FW is considered a measure of the potential of the dissolved factors to induce damage in the colonic mucosa. FW was extracted from the faecal matter of the rats and was applied in vitro to cell lines in culture. FW derived from prebiotic- or synbiotic-treated animals had a significantly lower genotoxic potential in human cell lines when compared to the FW from the control group. The genotoxic potential of FW was determined using the single cell gel electrophoresis or COMET assay (Klinder et al. 2004). The overall genotoxic potential of tumour-bearing rats was significantly higher than that of non-tumour-bearing rats, independent of the diet that was used. The correlation between genotoxic activity in faeces and tumour incidence indicates that reducing the genotoxicity of the faecal or caecal water might be another mechanism of chemoprevention of carcinogenesis. Prebiotic- and synbioticinduced colonic fermentation reduced the exposure of the gut mucosa to genotoxins.

In healthy rats (not treated with carcinogenic AOM), oligofructose-enriched inulin mainly interacted with the gut-associated lymphatic tissue. IL-10 production in the Peyer's patches was enhanced and the secretory $\operatorname{IgA}$ production was stimulated in the caecum as compared to the control group that received a standard, non-sup'plemented diet. Immune markers were not significantly modified in probiotic (LGG and Bb12)-fed animals. The synbiotic combination, however, increased secretory $\operatorname{IgA}$ production in the ileum and natural killer cell activity in peripheral blood, while it reduced the oxidative burst activity of blood neutrophils as compared to the probiotic-fed rats. The synbiotic combination of probiotics and prebiotics does not simply result in an additive effect (Roller et al. 2004a).

In a model in which carcinogenesis was induced with AOM, the functioning of the immune system was suppressed. When AOMtreated rats were fed a prebiotic (oligofructose-enriched inulin) or a synbiotic (oligofructose-enriched inulin + LGG and Bb12) the immunosuppressive effect of AOM in spleen, mesenteric lymph nodes and Peyer's patches was counterbalanced (levels became similar as in control group). No such effects were observed with the probiotic combination used in this study.

Prebiotic and synbiotic treatment reduced the tumour incidence (Femia et al. 2002). This anticarcinogenic effect could be correlated with a stimulation of natural killer cytotoxicity and increased IL-10 production in the intestine's Peyer's patches (Roller et al. 2004b).

\section{Human intervention study}

Design

The third and ultimate part of the SYNCAN project was the human intervention study - pilot study.

A common criticism of probiotic research is that the fate of the introduced bacterium in the gastrointestinal tract is not always investigated. It is important to confirm that introduced probiotic strains transit the gastrointestinal tract so that any observed effect may be associated with probiotic consumption. In the human intervention study subjects consumed wild-type probiotic

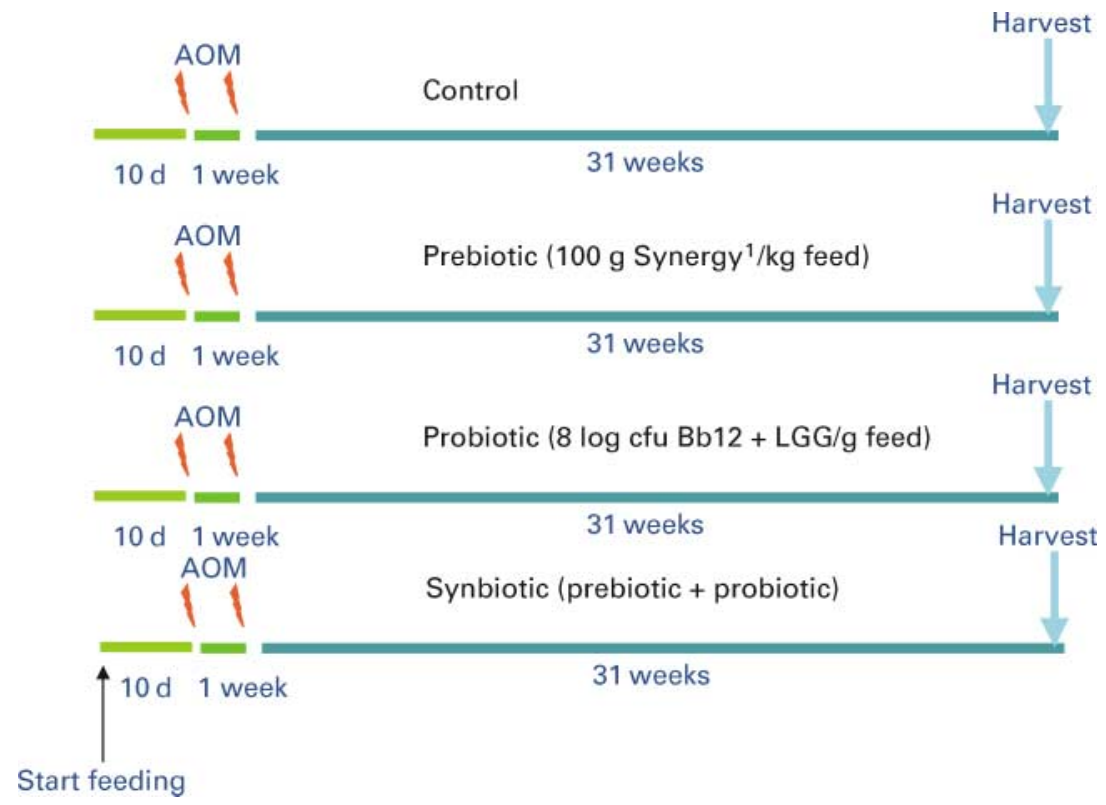

Fig. 1. Experimental design of the long-term chemoprevention anticancer model where rats ( $n$ 25) were injected twice with azoxymethane (AOM) with 1-week after a $10 \mathrm{~d}$ adaptation period to the diets. Colon tumour incidence is counted 31 weeks later. 
strains, which cannot be selectively isolated from the faeces. In order to confirm that LGG and Bb12 strains could survive the transit through the human gastrointestinal tract we performed a pilot study with antibiotic-resistant strains in three healthy volunteers.

Spontaneously occurring rifampicin-resistant $\left(\right.$ rif $^{\mathrm{R}}$ ) mutants were selected from Bb12 and LGG. Previous experience in humans demonstrated that such rif $^{\mathrm{R}}$ strains were not present in stool samples, and that rifampicin resistance is a suitable marker to allow selective isolation and enumeration of introduced probiotic strains from faeces (Dunne et al. 1999, 2001). The rifampicin-resistant strains were freeze-dried and the resultant freeze-dried product was checked for purity.

Briefly, a 1.51 fermenter containing modified MRS broth [0.4\% yeast extract, $2 \%$ glucose, $0.2 \% \quad \mathrm{~K}_{2} \mathrm{HPO}_{4}, 0.5 \%$ Na-acetate, $0.2 \%\left(\mathrm{NH}_{4}\right)_{3}$ citrate, $0.02 \% \mathrm{MgSO}_{4}, 0.0046 \% \mathrm{MnSO}_{4}$ and $1.8 \%$ peptone] for LGG rif $^{\mathrm{R}}$ or modified MRS broth containing $0.05 \%$ cysteine for Bb12 iif $^{\mathrm{R}}$ was inoculated overnight with cultures of rif $^{\mathrm{R}}$ strains. Fermentation was carried out for $16 \mathrm{~h}$ at $37^{\circ} \mathrm{C}$ and constant $\mathrm{pH}$ with stirring. The suspension was centrifuged, the pelleted bacteria resuspended in $10 \%$ skim milk, $2 \%$ sucrose and ascorbic acid and frozen at $-80^{\circ} \mathrm{C}$ for at least $1 \mathrm{~h}$. The bacteria were freeze-dried for $48 \mathrm{~h}$ and viable bacteria per gram of freeze-dried product were enumerated by spread plating appropriate dilutions on MRS agar (LGG) and modified raffinose bifidobacterium agar (Hartemink et al. 1996), where raffinose was replaced with lactose, for $\mathrm{Bb} 12$. The number of viable bacteria in the freeze-dried product was found to be $5.9 \times 10^{10} \mathrm{cfu} / \mathrm{g}$ in the case of Bb12 iif $^{\mathrm{R}}$ and $4.7 \times 10^{10} \mathrm{cfu} / \mathrm{g}$ in the case of LGG rif $^{\mathrm{R}}$. The freeze-dried rif $^{\mathrm{R}}$ strains and the wild-type parent strains were found to be identical using API (a rapid biochemical test).

In the SYNCAN project, it was considered desirable that the probiotics reach the colon in a viable state in order to exert possible effects. As the acidity in the stomach is a main reason for bacteria not to survive passage through the upper intestinal tract, the probiotics were administered in a coated capsule. The coating resists the acid conditions in the stomach, and only opens up in the small intestine. The selected probiotic strains have previously been shown to resist the bile acids that are secreted in the small intestine. The freeze-dried strains were therefore encapsulated and three healthy volunteers consumed one capsule containing $10^{10} \mathrm{cfu}$ of both LGG iff $^{\mathrm{R}}$ and Bb12 rif $^{\mathrm{R}}$ with a sachet of oligofructose-enriched inulin $(10 \mathrm{~g})$ for a
$7 \mathrm{~d}$ period. The volunteers provided faecal samples before feeding and at the end of feeding. The number of rif $^{\mathrm{R}}$ bacteria present in faeces before and after $7 \mathrm{~d}$ of feeding was enumerated using standard plate count techniques.

Total stool from one passage was collected in a plastic container and mixed using a sterile wooden spatula. An aliquot was used to make a faecal slurry with sterile $60 \% \mathrm{PBS} / 40 \%$ glycerol $/ 0.05 \%$ cysteine which was serially diluted 10 -fold in sterile PBS/0.05\% cysteine. Appropriate dilutions were spreadplated on the media described previously.

The goal was to recover at least $10^{5} \mathrm{cfu}$ of each strain/g of faeces, a level that was presumed to be the lowest active dose and to correspond to an acceptable $0.1 \%$ survival (oro-anal transit) of the bacteria based on the calculation that in volunteers receiving $10^{10} \mathrm{cfu} / \mathrm{d}$ and producing on average $100 \mathrm{~g}$ stool/d, $10^{8} \mathrm{cfu}$ would have to be recovered if the viability had been $100 \%$. In this study on average $4.17 \times 10^{6}$ and $2.65 \times 10^{7} \mathrm{cfu}$ of rif $^{\mathrm{R}} \mathrm{LGG}$ and rif $^{\mathrm{R}} \mathrm{Bb12}$, respectively, were recovered per $\mathrm{g}$ of faeces (Fig. 2) indicating that $1-10 \%$ of the probiotics survived passage through the gastrointestinal tract, which is considered a high survival rate. This experiment was carried out in the presence of the prebiotic. These results indicate that the probiotics when administered as a synbiotic combination are able to transit the human gastrointestinal tract and reach the colon in a viable state and in sufficient numbers to potentially exert a beneficial effect.

\section{Human intervention study}

A 12-week randomised, double-blind, placebo-controlled trial of a synbiotic food supplement for reduction in cancer risk biomarkers was carried out in The Mercy University Hospital, Cork, Ireland. The synbiotic product contained a probiotic mixture of L. rhamnosus GG (LGG) and B. lactis $\mathrm{Bb} 12$ (Bb12) and the prebiotic oligofructose-enriched inulin. This study was evaluated and approved by the Cork University Hospitals Ethics Committee and the subjects gave their informed consent.

A group of volunteers at increased risk for colon cancer (polypectomised) and (colon cancer subjects who had previously undergone 'curative resection' for colon cancer) were selected. A total of eighty subjects (fourty-three polyp, thirty-seven cancer) who fulfilled the inclusion and exclusion criteria (Table 1)

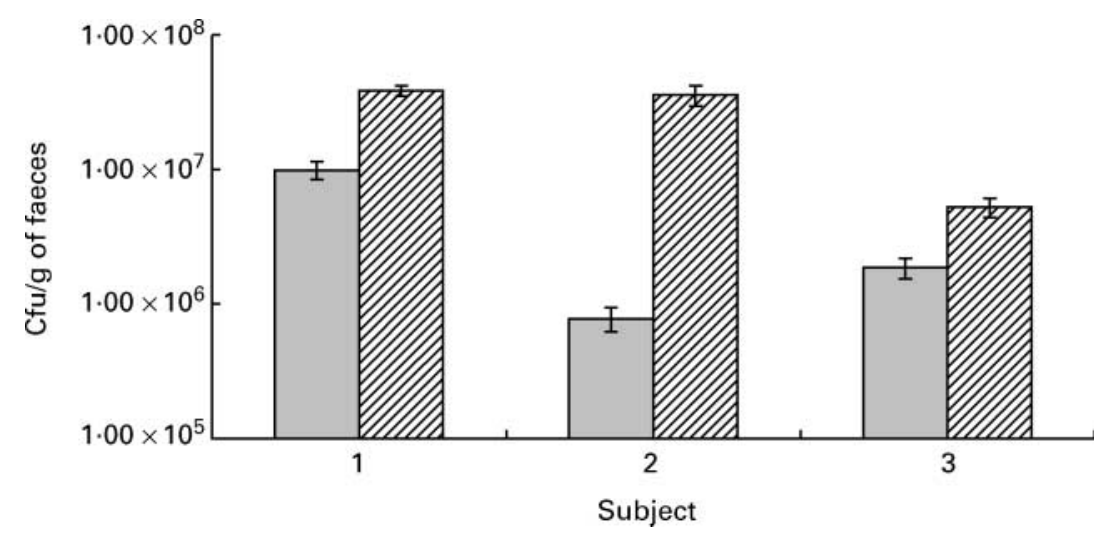

Fig. 2. Demonstration of the high viability of rifampicin-resistant Lactobacillus rhamnosus LGG ( $\square$ ) and Bifidobacterium lactis Bb12 (局) recovered from faeces in three healthy volunteers after daily consumption of $10^{10} \mathrm{cfu}$ of these bacteria for $7 \mathrm{~d}$. Mean values with standard error of the mean represented by veritcal bars. 
were recruited for the intervention. Overall nineteen cancer and twenty-one polyp volunteers received placebo (maltodextrin), while eighteen cancer and twenty-two polyp volunteers received the synbiotic combination. The outcome of the various mucosal, immunological and FW parameters will be reported elsewhere.

The outline of the study was as follows:

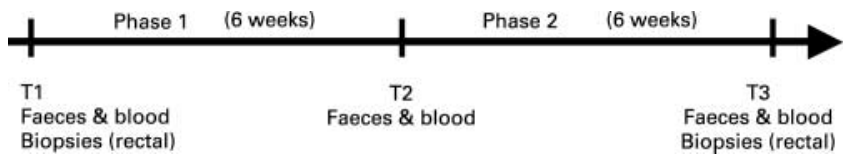

The subjects attended appointments with the study nurse at T1 (before intervention), T2 (after 6 weeks intervention) and T3 (after 12 weeks intervention). Blood and faecal samples were obtained at $\mathrm{T} 1, \mathrm{~T} 2$ and $\mathrm{T} 3$. Rectal biopsies were obtained by sigmoidoscopy performed by surgical registrars at $\mathrm{T} 1$ and T3. Subjects were randomly assigned to two groups. One group received the synbiotic product [encapsulated probiotic bacteria $\left(10^{10} \mathrm{cfu}\right.$ LGG and $\left.10^{10} \mathrm{cfu} \mathrm{Bb} 12\right)$ and a $10 \mathrm{~g}$ sachet of prebiotic oligofructose-enriched inulin]. The other group received the placebo product (encapsulated maltodextrin and a $10 \mathrm{~g}$ sachet of maltodextrin). Subjects kept a 6-week diary for each phase of the intervention. At $\mathrm{T} 1$ and $\mathrm{T} 2$ the subjects received a box containing sufficient product for 6 weeks. At T2 and T3 subjects were interviewed by the study nurse, and reactions to the product, medications taken and any adverse events, which had occurred in each 6-week period, were recorded.

Subjects provided samples at three time points. Faecal samples were provided at the beginning of intervention (week 0), midway through intervention (week 6) and at the end of intervention (week 12) for assessment of biomarkers and faecal flora. For the latter, total stool from one passage was collected in a plastic container and mixed using a sterile wooden spatula. An aliquot was used to make a faecal slurry with sterile $60 \%$ PBS $/ 40 \%$ glycerol $/ 0.05 \%$ cysteine. Faecal slurries were stored at $-80^{\circ} \mathrm{C}$ until analysis, thawed, and serially diluted 10 -fold

Table 1. Inclusion and exclusion criteria for human volunteers in the SYNCAN dietary intervention study

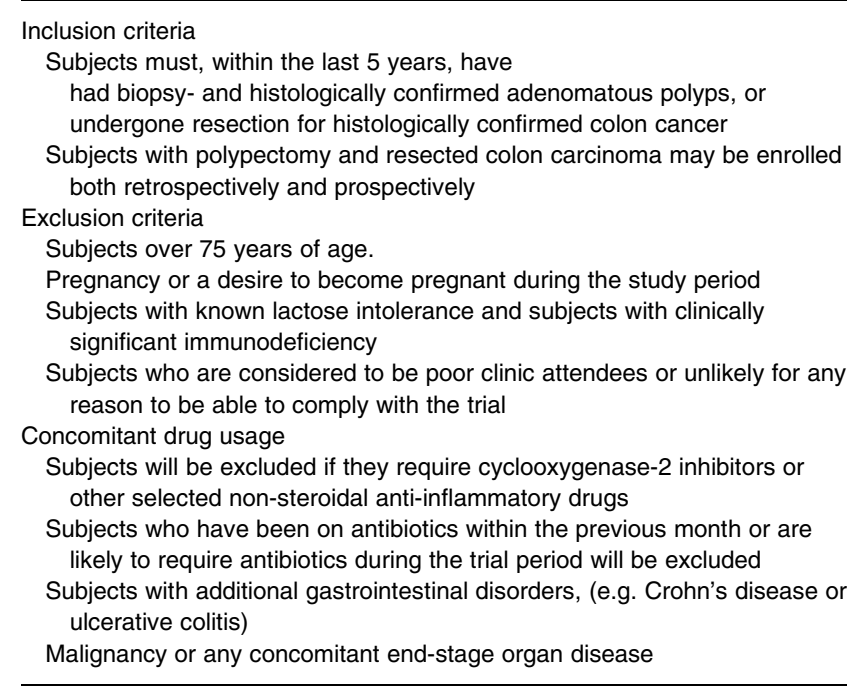

in sterile PBS/0.05\% cysteine and either spread-plated or pourplated in appropriate dilutions on suitable media. Colony identities were confirmed by Gram stain. Microbial numbers were calculated as colony forming units per gram of wet weight of faeces.

Blood samples were provided at the beginning of intervention (week 0), midway through intervention (week 6) and at the end of intervention (week 12) for assessment of immune markers and cholesterol measurements.

Rectal biopsy samples were taken at the beginning of intervention (week 0) and at the end of intervention (week 12) for investigation of genotoxic damage, mucosal cell proliferation and gene expression.

The study was carried out in a double-blinded manner. Subjects were coded from Syn-01 to Syn- 80 and each time point was coded $\mathrm{X}, \mathrm{Y}$ or $\mathrm{Z}$. Coded samples were processed and transported to partners involved in biomarker assessment in batches as subjects completed intervention to ensure that analysis of samples was also carried out in a blinded manner. For the duration of the study the code was stored by one individual and following completion of all analyses was distributed to all partners.

\section{Results}

Faecal flora. The ability of the synbiotic combination to modulate the gut flora was demonstrated in both the cancer and the polypectomised subjects (Fig. 4). The prebiotic effect was also confirmed as prebiotics have been reported to enhance the growth of bifidobacteria and other Gram-positive bacteria, while remaining unfermented by the majority of Gram-negative bacteria in the colon. The consumption of probiotics may also have contributed to the increase in bifidobacteria and lactobacilli. The viable faecal counts of the ingested probiotics $(5-7 \log \mathrm{cfu}$ ), however, were up to three orders of magnitude lower than the total Bifidobacterium and Lactobacillus count in the faeces of the volunteers $(8-10 \log \mathrm{cfu})$.

Bifidobacterium in both polypectomised and cancer patients consuming synbiotic significantly increased, as did the Lactobacillus in the polypectomised patients (Lactobacillus was increased at $\mathrm{T} 2$ in the cancer group although not significantly $P=0.067$ ), after a period of 6 weeks and of 12 weeks intervention (compared to T0 of each group). Previously, Kruse et al. (1999) reported that the prebiotic effect of inulintype fructans lasted for 2 months ( 9 weeks). In the control groups the bifidobacteria and lactobacilli either remained unaffected, or even decreased (cancer patients) during the intervention period. In the groups consuming synbiotics the benefit of consuming prebiotics and probiotics together was proven. The selectivity of fermentation, which is the main characteristic of the prebiotic effect, was shown by the bifidogenic effect, which was accompanied by a decrease of coliforms in both polypectomised and cancer patient groups and a decrease of (potentially pathogenic) Clostridium perfringens in the polypectomised group. The selectivity of the interaction of synbiotics is further demonstrated by the lack of effect on Bacteroides populations.

These results thus demonstrate that the synbiotic preparation administered to volunteers modulated the flora. Thus, it can be hypothesised that the eventual changes in biomarkers monitored in the human anticancer study in test groups $v$. control group 
(a)

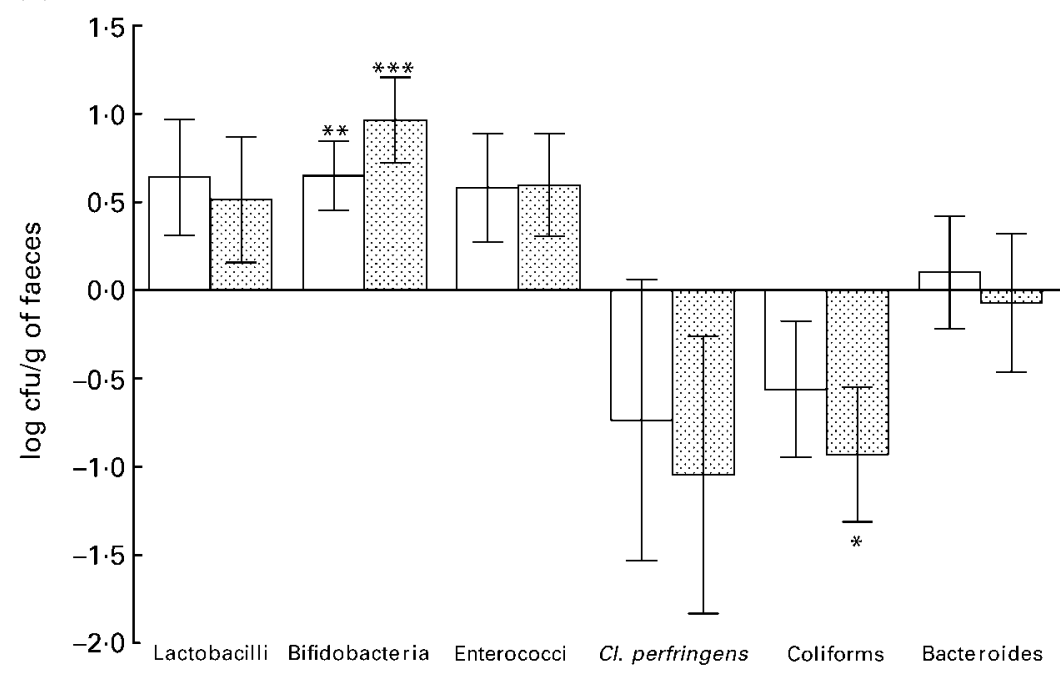

Bacterial group

(b)

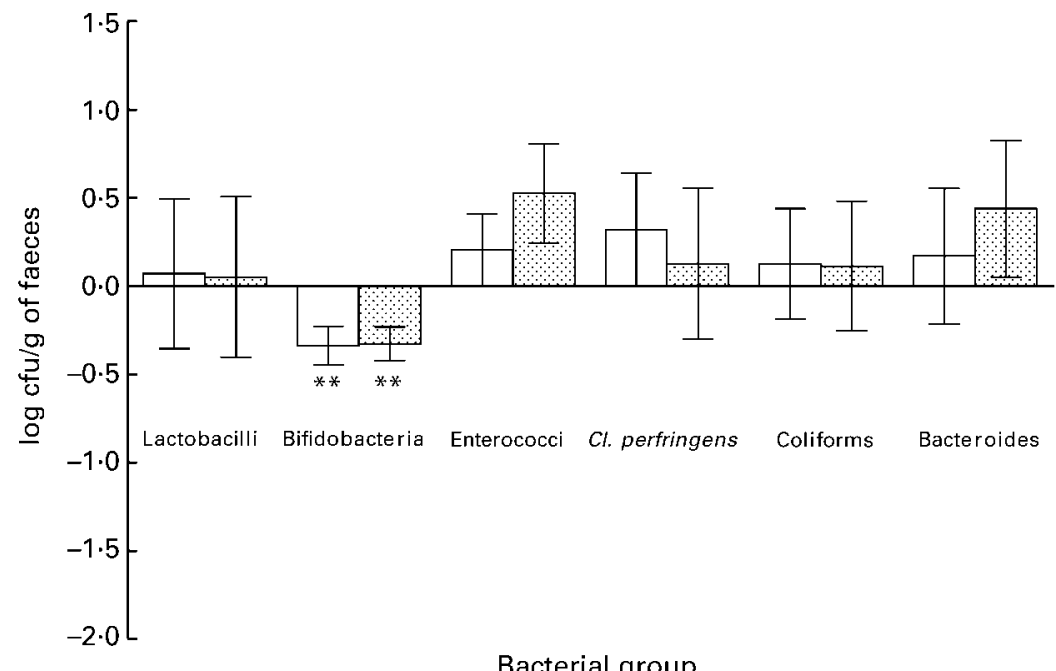

Bacterial group

Fig. 3. Demonstration of the efficacy of the synbiotic mixture to modulate the gut flora (a), compared to placebo (b), in cancer patients after 6 weeks ( $\square$ ) and 12 weeks (滕) of intervention compared to week 0 . Mean values with standard error of the mean represented by vertical bars. Bifidobacterium and Lactobacillus increased, Clostridium and coliforms decreased and Bacteroides were unaffected. Mean values were significantly different compared to week 0 : $\left({ }^{\star} P=0.01\right.$ to 0.05 , ${ }^{\star \star} P=0.001$ to $0.01,{ }^{\star \star \star} P<0.0001$.

are likely to be the consequence of the administration of an active synbiotic preparation.

\section{Discussion and conclusions}

The in vitro and in vivo animal studies have shown that the chosen synbiotic (Synergy1/LGG/Bb12) can modulate the genotoxic and cytotoxic potential of cell-free fermentation supernatants and reduce tumour incidence in AOM-induced carcinogenesis in rats. Moreover, the analysis of various markers showed reduced genotoxicity, increased caecal SCFA particularly butyrate, priming of the immune system to be more efficient to fight cancer cells and to counteract inflammation, and a reduction in DNA damage. These concurred with earlier reported anticancer activities of prebiotics, probiotics and synbiotics, and confirmed that the hypothesis 'are synbiotics relevant to fight cancer in human volunteers' could be investigated using the selected synbiotic preparation in a human dietary intervention study.

The description of the experimental design of the human study in the SYNCAN project shows that all conditions were met to generate objective results, in optimal conditions for the synbiotics (both probiotics and prebiotics were shown to be functional) to exert their possible anticancer effects. The results and the discussion of the results of the project are not fully completed at the time of publication of present paper. They will be published in the near future.

However, if the synbiotic approach is proven to suppress the carcinogenic process, then future research should focus on elucidating the effect of the prebiotic (oligofructose-enriched inulin or Synergy1) and the probiotics separately. Understanding these mechanisms will help in optimising the use of prebiotic, probiotic 
(a)

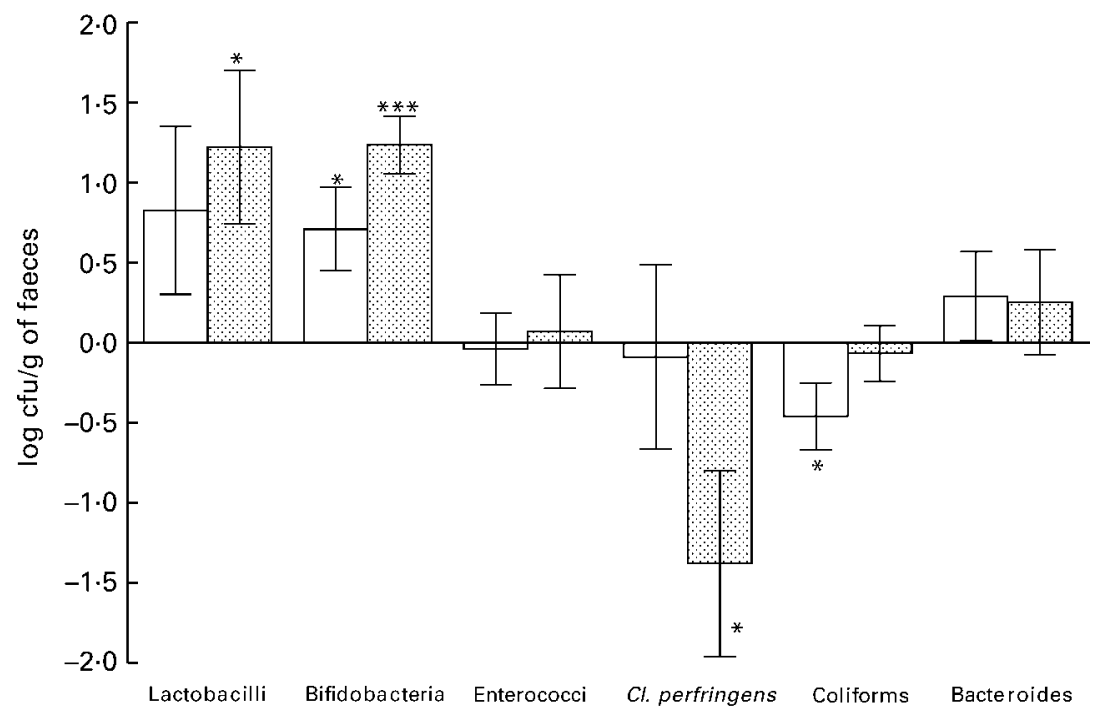

Bacterial Group

(b)

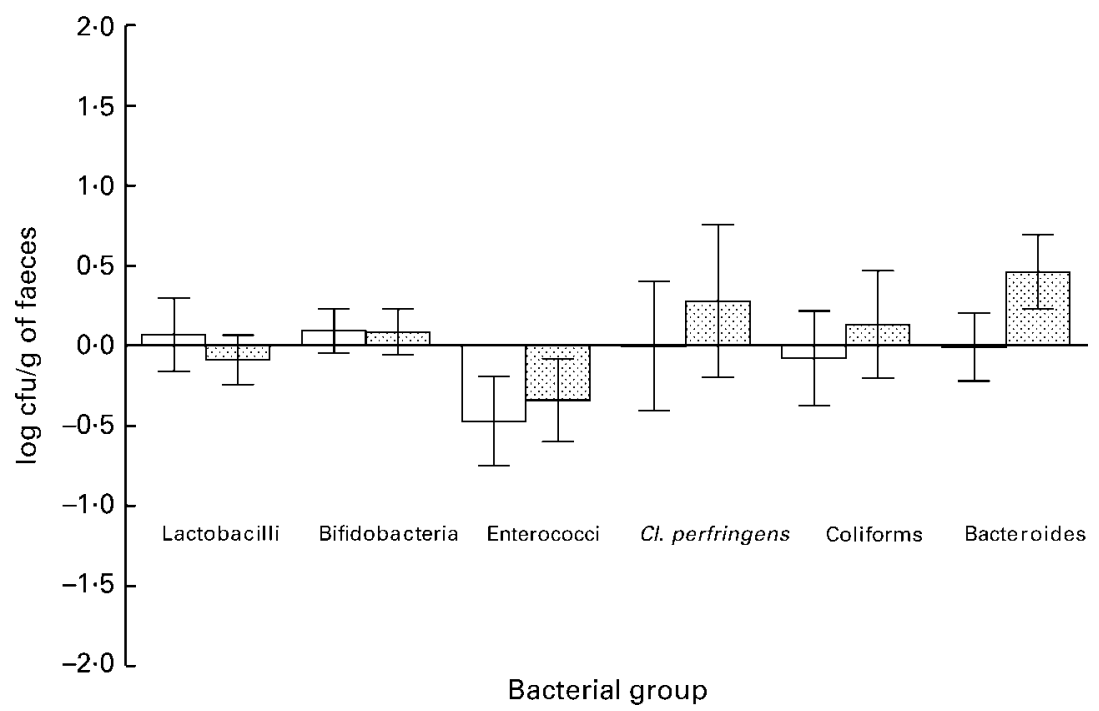

Fig. 4. Demonstration of the efficacy of the synbiotic mixture to modulate the gut flora (a), compared to placebo (b), in polypectomised patients after 6 weeks $(\square)$ and 12 weeks (圆) of intervention compared to week 0 . Mean values with standard error of the mean represented by vertical bars. Bifidobacterium and Lactobacillus increased, Clostridium and coliforms decreased and Bacteroides were unaffected. Mean values were significantly different compared to week 0 : ${ }^{*} P=0.01$ to $0.05 ;{ }^{* \star} P=0.001$ to $0.01 ;{ }^{* \star \star} P<0.0001$.

or synbiotic approaches to fight against cancer in early phases. To prevent is better than to heal.

\section{Acknowledgements}

This study project is being carried out with financial support of the European Communities, specific RTD programme 'Quality of Life and Management of Living Resources', Key Action 1 'Food, Nutrition and Health'. It does not necessarily reflect its views and in no way anticipates the Commission's future policy in this area. The authors would like to thank Claire Cronin, Dr Sean McCarthy, Dr Chris Collins, Dr Michael Moore, Professor Gerald O'Sullivan, Dr Michael Bennett, Mr Michael O'Riordan, Dr Colum Dunne,
Dr Jim Claire, Karen Meaney and Maurice O'Donoghue of the Mercy University Hospital, the Cork Cancer Research Centre and the Department of Microbiology, University College Cork for their help and contribution towards this research.

\section{References}

Bolognani F, Rumney CJ, Pool-Zobel BL \& Rowland IR (2001) Effect of lactobacilli, bifidobacteria and inulin on the formation of aberrant crypt foci in rats. Eur J Nutr 40, 293-300.

Dunne C, Murphy L, Flynn S, et al. (1999) Probiotics: from myth to reality. Demonstration of functionality in animal models of disease and in human clinical trials. Antonie Van Leeuwenhoek 76, 279-292. 
Dunne C, O’Mahony L, Murphy L, et al. (2001) In vitro selection criteria for probiotic bacteria of human origin: correlation with in vivo findings. Am J Clin Nutr 73, Suppl., 386S-392S.

Femia AP, Luceri C, Dolara P, Giannini A, Biggeri A, Salvadori M, Clune Y, Collins KJ, Paglierani M \& Caderni G (2002) Antitumorigenic activity of the prebiotic inulin enriched with oligofructose in combination with the probiotics Lactobacillus rhamnosus and Bifidobacterium lactis on azoxymethane-induced colon carcinogenesis in rats. Carcinogenesis 23, 1953-1960.

Gallaher DD, Stallings WH, Blessing LL, Busta FF \& Brady LJ (1996) Probiotics, cecal microflora, and aberrant crypts in the rat colon. $J$ Nutr 126, 1362-1371.

Hartemink R, Kok BJ, Weenk GH \& Rombouts FM (1996) Raffinose-Bifidobacterium (RB) agar, a new selective medium for bifidobacteria. $J$ Microbiol Methods 27, 33-43.

Klinder A, Förster A, Caderni G, Femia AP \& Pool-Zobel BL (2004) Fecal water genotoxicity is predictive of tumor preventive activities by inulin-like oligofructoses, probiotics (Lactobacillus rhamnosus and Bifidobacterium lactis) and their synbiotic combination. Nutr Cancer 49, 144-155.

Klinder A, Gietl E, Hughes R, Jonkers N, Pistoli S, Gibson G, Rowland I, Van Loo J \& Pool-Zobel B (2003) Gut fermentation products of inulinderived prebiotics inhibit markers of tumor progression in human colon tumor cells. J Cancer Prev 1, 19-32.

Kruse HP, Kleessen B \& Blaut M (1999) Effects of inulin on faecal bifidobacteria in human subjects. Br J Nutr 82, 375-382.

Kulkarni N \& Reddy BS (1994) Inhibitory effect of Bifidobacterium longum cultures on the azoxymethane-induced aberrant crypt foci formation and fecal bacterial beta-glucuronidase. Proc Soc Exp Biol Med 207, 278-283.

McBain AJ \& Macfarlane GT (1997) Investigations of bifidobacterial ecology and oligosaccharide metabolism in a three-stage compound continuous culture system. Scand J Gastroenterol Suppl 22, 232-240.

O’Mahony L, Feeney M, O'Halloran S, Murphy L, Kiely B, Fitzgibbon J, Lee G, O’Sullivan G, Shanahan F \& Collins JK (2001) Probiotic impact on microbial flora, inflammation and tumour development in IL-10 knockout mice. Aliment Pharmacol Ther 15, 219-225.

Pierre F, Perrin P, Champ M, Bornet F, Meflah K \& Menanteau J (1997) Short-chain fructo-oligosaccharides reduce the occurrence of colon tumors and develop gut-associated lymphoid tissue in Min mice. Cancer Res 57, 225-228.

Rao VA (2001) The prebiotic properties of oligofructose at low intake levels. Nutr Res 21, 843-848.
Reddy BS, Hamid R \& Rao CV (1997) Effect of dietary oligofructose and inulin on colonic preneoplastic aberrant crypt foci inhibition. Carcinogenesis 18, 1371-1374.

Roller M, Rechkemmer G \& Watzl B (2004a) Prebiotic inulin enriched with oligofructose in combination with the probiotics Lactobacillus rhamnosus and Bifidobacterium lactis modulates intestinal immune functions in rats. $J$ Nutr 134, 153-156.

Roller M, Femia AP, Caderni G, Rechkemmer G \& Watzl B (2004b) Intestinal immunity of rats with colon cancer is modulated by oligofructose-enriched inulin combined with Lactobacillus rhamnosus and Bifidobacterium lactis. Br J Nutr 92, 931-938.

Rowland IR, Rumney CJ, Coutts JT \& Lievense LC (1998) Effect of Bifidobacterium longum and inulin on gut bacterial metabolism and carcinogen-induced aberrant crypt foci in rats. Carcinogenesis 19, 281-285.

Salminen S, Bouley C, Boutron-Ruault MC, Cummings JH, Franck A, Gibson GR, Isolauri E, Moreau MC, Roberfroid M \& Rowland I (1998) Functional food science and gastrointestinal physiology and fucntion. Br J Nutr 80, Suppl. 1, S147-S171.

Taper HS, Lemort C \& Roberfroid MB (1998) Inhibition effect of dietary inulin and oligofructose on the growth of transplantable mouse tumor. Anticancer Res 18, 4123-4126.

Taper HS \& Roberfroid M (1999) Influence of inulin and oligofructose on breast cancer and tumor growth. J Nutr 129, Suppl., 1488S-1491S.

Taper HS \& Roberfroid MB (2000) Inhibitory effect of dietary inulin or oligofructose on the development of cancer metastases. Anticancer Res 20, 4291-4294.

Van Loo J (2004) The specificity of the interaction with intestinal fermentation by prebiotics determines their physiological efficacy. Nutr Res $\operatorname{Rev~17,89-98.~}$

Van Loo J \& Jonkers N (2001) Evaluation in human volunteers of the potential anticarcinogenic activities of novel nutritional concepts: prebiotics, probiotics and synbiotics (the SYNCAN project QLK1-199900346). Nutr Metab Cardiovasc Dis 11, 87-93.

Verghese M, Rao DR, Chawan CB, Williams LL \& Shackelford LA (2002) Dietary inulin suppresses azoxymethane-induced aberrant crypt foci and colon tumors at the promotion stage in young Fisher 344 rats. J Nutr 132, 2809-2813.

Verghese M, Walker LT, Shackelford LA, Chawan CB \& Van Loo J (2003) Inhibitory effects of non-digestible carbohydrates of different chain lengths on AOM induced aberrant crypt foci in Fisher 344 rats. In Proccedings of the Second Annual AACR International Conference. Frontiers in Cancer Prevention Research, Phoenix, AZR, 26-30 October 2003. Poster B186 Abstr. 